\title{
L'HOMME L'Homme
}

Revue française d'anthropologie

239-240 | 2021

Expériences initiatiques du genre

Denis-Constant Martin, Plus que de la musique... Musiques, sociétés et politique, Caraibes, États-Unis, Afrique du Sud

\section{Maël Péneau}

\section{OpenEdition}

Journals

Édition électronique

URL : https://journals.openedition.org/lhomme/41369

DOI : 10.4000//homme.41369

ISSN : 1953-8103

Éditeur

Éditions de l'EHESS

Édition imprimée

Date de publication : 1 décembre 2021

Pagination : 342-344

ISBN : 978-2-7132-2868-1

ISSN : 0439-4216

Référence électronique

Maël Péneau, «Denis-Constant Martin, Plus que de la musique... Musiques, sociétés et politique, Caraibes, États-Unis, Afrique du Sud », L'Homme [En ligne], 239-240 | 2021, mis en ligne le 01 décembre 2021, consulté le 15 décembre 2021. URL : http://journals.openedition.org//homme/41369; DOI : https:// doi.org/10.4000//homme.41369

Ce document a été généré automatiquement le 15 décembre 2021.

(c) École des hautes études en sciences sociales 


\title{
Denis-Constant Martin, Plus que de la musique... Musiques, sociétés et politique, Caraibes, États-Unis, Afrique du Sud
}

\author{
Maël Péneau
}

\section{RÉFÉRENCE}

Denis-Constant Martin, Plus que de la musique... Musiques, sociétés et politique, Caraibes, États-Unis, Afrique du Sud. Guichen, Mélanie Seteun, 2020, 550 p., bibl., ill. (« Musique et société »).

$1 \quad$ Plus que de la musique rassemble dix-sept textes publiés par Denis-Contant Martin entre 1990 et 2017, et donne ainsi au lecteur l'occasion de mesurer le rôle fondamental joué par ce chercheur, sociologue et politologue, aujourd'hui directeur de recherche émérite à Science-Po, dans le développement et l'évolution de la sociologie des musiques populaires. Ce faisant, cette compilation met en lumière les processus à travers lesquels cette discipline, relativement récente en France, contribue à la compréhension de phénomènes et d'enjeux majeurs de nos sociétés contemporaines, au Nord comme au Sud. Puisant dans les sciences politiques, la sociologie des sociétés africaines, l'ethnomusicologie, les études sur le jazz, mais aussi dans les études postcoloniales, cet ouvrage nous donne enfin à voir un parcours d'une grande liberté intellectuelle, jalonné de rencontres inspirantes, de Georges Balandier à Simha Arom, en passant par Édouard Glissant ou Didier Levallet.

2 La première partie porte sur les différentes approches et méthodes relevant de l'analyse des musiques dites "populaires", terme dont la définition problématique incite Denis-Constant Martin à lui préférer celle de « musiques de masse ", empruntée à l'historien Jean-François Sirinelli ${ }^{1}$. Bien que découlant nécessairement de l'objet de la 
recherche et de la problématique, le choix de la méthodologie est crucial car il doit conduire à faire dialoguer l'ethnographie, l'analyse musicale, l'analyse des paroles, l'analyse symbolique ou des approches sémiotiques. Pour l'auteur, l'étude des musiques de masse a donc vocation à s'inscrire dans une musicologie générale, ainsi que dans le champ de l'ethnomusicologie, mais à la condition de développer une démarche résolument interdisciplinaire. Il s'agit ainsi d'appréhender «l'ensemble des pratiques musicales comme activités productives donnant du sens aux objets sonores » (p. 63) par l'analyse tout à la fois de leurs contextes, des mécanismes de production et des formes de la réception, ou encore en cherchant à mieux comprendre les objets musicaux euxmêmes. Mais la musique de masse est toujours "plus que de la musique", rappelle Denis-Constant Martin. En tant que fait social, elle constitue un véritable objet d'étude qui permet d'entrer dans les sociétés contemporaines et d'en donner une lecture de l'ordre du sensible. De sorte que le travail du chercheur n'a pas pour vocation d'émettre un jugement de valeur sur la musique elle-même, mais d'examiner les liens complexes entre la façon dont elle est créée, composée, jouée ou écoutée, ainsi que les groupes sociaux au sein desquels s'inscrivent ces pratiques.

3 La question des sources et des ressources de la création musicale constitue l'un des thèmes qui jalonnent les travaux présentés dans cet ouvrage. Denis-Contant Martin interroge ainsi la notion d'« appropriation » à partir du contexte, des médiations, des processus, des significations variées qui lui sont données, comme des restrictions auxquelles elle peut être soumise. Il choisit d'analyser l'appropriation du point de vue de la création musicale elle-même, en mettant en évidence des dynamiques liées au "rafraîchissement du préexistant» (p.115), ou aux dimensions discursives des pratiques de réappropriation. En insistant sur le caractère plastique et évolutif de cette notion et de ses significations musicales, l'auteur articule sa réflexion à la question des identités. Il revient ainsi sur la musique comme moyen d'accéder aux représentations sociales «du soi et de l'autre»(p.139), mais aussi de renforcer les sentiments d'appartenance, ce qui le conduit à questionner les conditions d'émergence de la musique dans sa dimension politique. Dans le prolongement du travail engagé en 2002 autour des "objets politiques non identifiés ${ }^{2}$, c'est en s'intéressant aux aspects discursifs des expressions musicales, à leurs dimensions symboliques, à l'innovation ou à la transgression qu'elles produisent et aux imaginaires qu'elles suscitent, que DenisConstant Martin parvient à montrer comment la musique est un objet éminemment politique.

4 Dans la deuxième partie de l'ouvrage, Denis-Constant Martin emprunte à Édouard Glissant ${ }^{3}$ le concept de "créolité » pour engager une réflexion sur les transformations qui ont permis l'avènement de musiques nouvelles dans les sociétés esclavagistes, sur les conditions de la création en situation de domination et, enfin, sur les phénomènes issus de la globalisation. À partir des musiques afro-américaines, des spectacles de blackface minstrels ${ }^{4}$ et des chœurs malais du Cap en Afrique du Sud, l'auteur propose de définir la créolisation comme un processus plutôt que comme un état. Il écarte ainsi toute velléité de lecture essentialisante sur les sociétés étudiées, pour analyser en détail les multiples combinaisons possibles de métissage qui donnent naissance à des pratiques innovantes et à des expressions singulières. Mais l'auteur n'oublie jamais de montrer de quelles façons ces musiques, si créatives soient-elles, trouvent leurs sources dans des contextes marqués par la violence et la domination. 
Dans la dernière partie de l'ouvrage, plusieurs études de cas prolongent et illustrent les propositions épistémologiques et méthodologiques développées dans les chapitres précédents. Denis-Constant Martin s'intéresse ainsi aux significations sociales et politiques des musiques de masse en s'appuyant sur l'exemple des musiques jamaïcaines, des spirituals afro-américains, ou encore des musiques trinidadiennes. Il y examine "l'impact de la protestation symboliquement inscrite dans des chansons (paroles et musiques) sur les attitudes et comportements politiques des citoyens " (p. 192), tout en refusant d'en faire une lecture qui assignerait les expressions musicales aux seules fonctions de marqueurs des conditions et des évolutions politiques et socioéconomiques. Quatre chapitres témoignent de l'intérêt profond de l'auteur pour les musiques et les cultures sud-africaines et de sa connaissance extrêmement fine de l'Afrique du Sud post-apartheid. Dans le chapitre XI, il aborde la diversité du jazz sudafricain, témoin de la multiplicité des identités locales et des mécanismes de créolisation constitutifs de la société sud-africaine. Dans le chapitre suivant, il interroge la perception que les Afrikaners nés après l'apartheid ont de leur situation et de celle du pays à travers un clip vidéo et ses significations ambivalentes et contradictoires. L'auteur se penche aussi sur l'évolution des conceptions esthétiques mobilisées par les malay choirs $^{5}$ et les compositeurs de moppies ${ }^{6}$ du Cap, en mettant la focale sur les processus de construction des identités. Articulant l'approche sociologique à l'approche musicologique, un texte coécrit avec l'ethnomusicologue Simha Arom vient clore cet ouvrage par la question des «musiques du monde». À partir des conditions de leur "invention", de leur succès et de l'analyse de leur "fonctionnement», qui résultent de combinaisons multiples entre des éléments préexistants, tous deux montrent combien ces musiques éclairent les processus à l'œuvre dans la vaste entreprise de globalisation du monde.

Marqués par la transversalité et la pluralité des cas étudiés, les travaux réunis dans cet ouvrage permettent de mettre en lumière les thématiques et les réflexions qui jalonnent le parcours scientifique de Denis-Constant Martin, et de mieux saisir sa portée et sa singularité dans le champ des travaux sociologiques sur les musiques populaires. La perspective transdisciplinaire et résolument ancrée dans le monde contemporain, qu'il a été l'un des premiers à défendre, donne ainsi des clés essentielles pour comprendre des phénomènes liés à la circulation, à l'apparition et à la création de musiques qui, jusque dans les années 1990, n'étaient que rarement considérées comme des objets de recherche légitimes. En ce sens, Denis-Constant Martin a contribué de manière déterminante à un tournant épistémologique majeur, qui a permis un renouvellement des approches en ethnomusicologie et en anthropologie de la musique, et l'émergence d'une nouvelle génération de chercheurs.

\section{NOTES}

1. Cf. Jean-François Sirinelli \& Jean-Pierre Rioux, eds, La Culture de masse en France de la Belle Époque à aujourd'hui, Paris, Fayard, 2002. 
2. Cf. Denis-Constant Martin, ed., Sur la piste des OPNI (objets politiques non identifiés), Paris, Karthala, 2002 («Recherches internationales »).

3. Cf. Édouard Glissant, L’Intention poétique, Paris, Le Seuil, 1969 (« Pierres vives »).

4. Spectacles apparus au début du XIX ${ }^{e}$ siècle aux États-Unis, à l'occasion desquels des hommes blancs, grimés en Noirs, proposent un répertoire stéréotypé et racialisé de musiques, danses et histoires attribuées à la communauté afro-américaine. Cf. William T. Lhamon, Peaux blanches, masques noirs. Performances du blackface, de Jim Crow à Michael Jackson, Paris, Kargo \& l'Éclat, 2008 [1998]. Voir également Nick Tosches, Blackface. Au confluent des voix mortes, Paris, Allia, 2003 [2001].

5. Également dénommés Nagtroepe («troupes de la nuit»), ces chœurs sont composés d'hommes majoritairement musulmans, classés durant la période de l'apartheid dans la catégorie "métis ». Le soir de la Saint-Sylvestre, ils défilent dans certains quartiers du Cap, accompagnés de musiciens (guitare, banjo, tambour, violoncelle). Cf. DenisConstant Martin, « Musique dans la rue et contrôle de l'espace urbain : le Cap (Afrique du Sud) », Cahiers internationaux de sociologie, 2005, 119 (2) : 247-265 (en ligne : https:// www.cairn.info/revue-cahiers-internationaux-de-sociologie-2005-2-page-247.htm).

6. Chansons sud-africaines constituées d'éléments empruntés à la variété internationale, au jazz et aux comédies musicales, combinés entre eux et réassemblés à l'aide de transitions originales adaptées au tempo et à la structure rythmique d'une forme musicale locale jouée lors de parades. 